\begin{abstract}
The Determinants of Salary and Bonus for Rank and File Employees

The current study integrates the repeated game approach to implicit contracts and the analysis of explicit bonus rules based on subjective performance evaluation to determine the optimal structure of the compensation scheme for the average whitecollar employee. In contrast to previous contributions we assume that the agent is risk-neutral but liquidity constrained. The salary reflects the maximum reward associated with contractual compliance which can be implicitly agreed. If the probability that the employee remains within the firm increases, the principal can reduce the rent captured by the agent. Hence, the optimal salary increases and the bonus decreases. Nevertheless, the optimal effort supply unambiguously increases.
\end{abstract}

Keywords: White-Collar Employees, Implicit Contract, Explicit Bonus, Reputational Equilibrium.

JEL-Classification: M52 - Compensation and Compensation Methods and Their Effects 


\section{The Determinants of Salary and Bonus for Rank and File Employees}

\author{
Dominique Demougin \\ School of Business and Economics \\ Humboldt University at Berlin*
}

\author{
Oliver Fabel \\ Department of Economics \\ University of Konstanz ${ }^{\dagger}$
}

August 2, 2004

${ }^{*}$ Prof. Dominique Demougin, PhD, Walter Rathenau Chair in Organization Theory, School of Business and Economics, Humboldt University at Berlin, Spandauer Str. 1, 10178 Berlin, Germany; Fone: +49-(0)30-2093-1341; Fax: +49-(0)30-2093-1343; E-Mail: dominique@demougin.de.

${ }^{\dagger}$ Prof. Dr. Oliver Fabel, Chair for Managerial Economics, in particular Business Policy, Department of Economics, Faculty of Law, Economics, and Public Administration, University of Konstanz, Box D144, 78457 Konstanz, Germany; Fone: +49-(0)7531-882990/-2992; Fax: +49-(0)7531-88-4456; E-Mail: oliver.fabel@uni-konstanz.de. 


\section{Introduction}

The current study investigates the optimal contract between a risk-neutral principal and an agent who is also risk-neutral but liquidity constrained. Although both parties can observe the agent's contribution to firm value, lack of verification precludes that the contract can be conditioned on this observation. However, given that the contractual environment reflects a repeated game, the principal can offer a self-enforcing implicit contract. In addition, he has invested in a monitoring technology which generates an unbiased performance signal. This signal is verifiable and can therefore be used to condition an explicit bonus scheme.

Any incentive contract which relies on such an explicit bonus is shown to induce a positive rent captured by the agent. This rent can be reduced by increasing the implicit reward for contractual compliance. Consequently, the principal will offer the maximum implicit reward which is still credible given that the contract with purely explicit effort incentives constitutes his outside option. The resulting optimal contract then consists of both an implicit reward and an explicit bonus scheme. The former can be interpreted as the agent's salary since it is constant over all periods as long as the agent complies with the implicit contract.

Comparative static analysis of the optimal contract allows to investigate the interplay of these salary and bonus components of the agent's compensation package. As should be expected, the salary is increased and the explicit performance bonus reduced if the probability that the agent leaves the firm in an upcoming period decreases. However, the optimal effort level ambiguously increases. The enhanced possibility to provide implicit reputational incentives more than compensates the rent savings effect associated with lowering the explicit bonus.

Economically, the contractual environment analyzed below applies to rank and file managers or average white-collar employees who supply internal services. In contrast, most of the theoretical and empirical work on management incentives has so far focussed on the problem of aligning top management incentives to serve the interests of firm owners. Notwithstanding that the solution to this problem involves a complex mix of various types of incentive pay - such as stock grants, stock option plans, and bonus thresholds defined conditional on the available accounting information - the manager's contribution to firm value always constitutes a contractible 
performance measure in these cases. However, according to Prendergast (1999), most white-collar jobs in firms lack such possibility of measuring performance by the effect of effort on firm value. ${ }^{1}$

Empirically, Aggarval and Samwick (2003) investigate the performance pay incentives for different groups of top managers defined according to their responsibility for corporate success. It is shown that the relationship between increases in shareholder wealth and the managers' performance pay is strongest for CEOs. Yet, there also exists a positive and significant pay-firm-performance sensitivity in groups of managers whose performance is not exclusively measured by their contribution to shareholder wealth. Rayton (2003) further reports that the pay-firm-performance elasticity of the average employee approximately matches the respective elasticities found in previous studies on CEO-incentives.

Such employees' bonus schemes are typically contingent on subjective performance measures. ${ }^{2}$ MacLeod (2003) then shows that subjective performance measurement generally implies more compressed compensation relative to the case with verifiable objective performance measures. However, while this study assumes that both contracting parties only possess private measures of performance which may be correlated, the standard approach to incentive setting under subjective performance evaluation uses the repeated game approach to derive the optimal self-enforcing contract. In this scenario the two parties possess identical beliefs concerning the subjective evaluation. ${ }^{3}$

The current model is most closely related to Baker et al. (1994) and Pearce and Stacchetti (1998) both of whom analyze the interplay between implicit contractual rules and explicit bonus schemes based on subjective performance evaluations. Given that the performance measure is unbiased, the former find that the implicit reward for contractual compliance and the explicit bonus are substitutes. If either the

\footnotetext{
${ }^{1}$ Also, there do not exist objective output measures to define piece-rates or similar incentive schemes associated with traditional blue-collar work.

${ }^{2}$ See Baker et al. (1994) and MacLeod and Parent (1999). Moreover, Ittner et al. (2003) find that the subjectivity of the performance evaluation increases over time if the evaluation process allows for sufficient discretion of the evaluator. In particular, they investigate the bonus awards in a large financial service firm which uses the Balanced Scorecard system for performance measurement.

${ }^{3}$ See Bull (1987), MacLeod and Malcolmsen (1989), and, most recently, Levin (2003) who shows that in this case the optimal contract should be of the shirk-threat type.
} 
implicit contract can nearly achieve the first-best or if performance measurement is sufficiently accurate, only one of these incentive mechanisms will be implemented by the optimal contract.

In contrast, Pearce and Stacchetti (1998) show that, accounting for learning from past observations, the two mechanisms are always complements. If the agent's performance history suggests that the income level in the next upcoming period should be high, the implicit reward increases and the explicit bonus decreases. This adjustment reflects optimal income-smoothing over time while maintaining the effectiveness of the effort incentives. Since the implicit contractual reward only depends on whether the employee has supplied the required effort level and is independent of firm performance, it can be associated with a fixed salary component.

Both Baker et al. (1994) and Pearce and Stacchetti (1998) require risk-aversion on the side of the employee in order to motivate the simultaneous use of implicit and explicit effort incentives. The respective conclusions then further depend on the stochastic properties of the performance signal. Investigating the interplay between implicit and explicit incentives with a risk-neutral but liquidity constrained employee, the current study therefore identifies yet a third and qualitatively different mechanism affecting the structure of the compensation package. A higher salary reflecting an enhanced possibility to provide implicit incentives serves to minimize the employee's rent derived from the explicit contract.

The remainder of the paper is organized as follows. Next section introduces the model framework. Section 3 provides the solution to the principal's sub-problem of minimizing the compensation costs associated with particular effort supplies of the agent. In section 4 we derive the optimal contract given that, in every future period, the principal can only offer a contract with purely explicit incentives if she breaches the implicit contract. The final section contains a summarizing discussion.

\section{The model}

We analyze a contracting problem between a risk-neutral principal and a risk-neutral agent who is liquidity-constrained in a stochastically repeated environment. Specifically, after each period the game is repeated next period with probability $p$. The 
agent leaves the firm and the game ends with probability $(1-p)$. For parsimony, we assume that there is no discounting.

In any given period if production takes place the agent supplies a productive effort $e \in[0,1]$ which generates value $v(e)$ with $v_{e}(e)>0$ and $v_{e e}(e) \leqq 0$. The agent's effort can be thought of as an internal service such that $e$ and $v(e)$ are nonverifiable by a third party. Consequently, they are not explicitly contractible. The agent's private costs of effort are given by $c(e)$ with $c(0)=c_{e}(0)=0, c_{e}(e)>0$, $c_{e e}(e)>0$, and $c_{e e e}(e) \geqq 0$ for $e>0$, and $\lim _{e \rightarrow 1} c(e)=+\infty{ }^{4}$

The principal is assumed to observe the agent's effort $e .^{5}$ Moreover, there is a monitoring technology generating a verifiable binary signal $s$ with $s \in\{0,1\}$. For parsimony, we assume $\operatorname{Pr}[s=1 \mid e]=e$ - hence, we measure effort in terms of the probability to observe the favorable signal.

Due to the repeated game structure, reputational equilibrium may allow the principal to make a credible promise even though it cannot be enforced by a third party. Therefore, the contract offered to the agent may specify an implicit reward scheme

$$
\hat{w}(e, \bar{e})=\left\{\begin{array}{c}
w \text { if } e \geqq \bar{e} \\
0 \text { if } e<\bar{e}
\end{array},\right.
$$

where $w \geqq 0$ denotes the agent's salary and $\bar{e}$ a threshold effort. In addition, the principal may offer an explicit bonus scheme

$$
\hat{b}(s)=\left\{\begin{array}{l}
b \text { if } s=1 \\
0 \text { if } s=0
\end{array},\right.
$$

contingent on the verifiable signal $s$ and enforceable by a court.

The reputational equilibrium will be sustained by a tit-for-tat strategy where, if the principal once breaks trust, the agent never believes him in all future periods to pay an implicit reward. In such a case, the fall-back contract would solely depend on the explicit bonus scheme. ${ }^{6}$ Thus, after a deviation the principal's maximum

\footnotetext{
${ }^{4}$ The main advantage of requiring a positive third derivative is to ensure that the principal's cost function of implementing effort $e$ is convex everywhere.

${ }^{5}$ Equivalently she can infer $e$ from $v(e)$.

${ }^{6}$ More generally, one would need to consider the possibility of a fixed payment $F$. If this payment is not bounded, it is well known from the literature that the first-best is attainable. However, since
} 
profit is

$$
\begin{gathered}
\pi^{D}=\max _{e, b} v(e)-b e \\
\text { s. t. } e=\arg \max _{\tilde{e} \geqq 0} b \tilde{e}-c(\tilde{e}) \\
b e-c(e) \geqq 0
\end{gathered}
$$

where the first constraint is a standard incentive compatibility condition and the second requirement follows from voluntary participation. The agent's first-order condition is sufficient and defines the effort implementable by the bonus $b$. Due to the shape of the agent's cost function, it is easily verified that the participation constraint is always satisfied, i.e. the agent receives a rent defined by $R(e) \equiv$ $c_{e}(e) e-c(e)$.

An illustrative way of restating the principal's maximization problem (I) is

$$
\pi^{D}=\max _{e} v(e)-c(e)-R(e)
$$

where $c(e)+R(e)$ are the principal's costs of implementing effort $e$. Given the foregoing assumptions, an interior solution always exists and it is unique. The optimal effort supply in the deviation contract, hereafter denoted by $e^{D}$, is characterized by the first-order condition

$$
v_{e}\left(e^{D}\right)-c_{e}\left(e^{D}\right)-c_{e e}\left(e^{D}\right) e^{D}=0
$$

Next, consider the case of designing a credible contract containing both implicit and explicit performance pay. Ex-post the principal will be tempted to deviate, thereby saving $w$. However, deviations are costly since afterwards per-period profits are constrained by $\pi^{D}$. Thus, the principal faces a trade-off resulting in a maximally credible implicit reward denoted by $\bar{w}$. Initially, taking $\bar{w}$ as given, we define

we are assuming that the agent is liquidity constrained, $F, F+b \geqq 0$. It is easily verified that, under the assumptions of the model, the optimal fixed payment $F$ is equal to zero. See Demougin and Fluet (2001). 
$C^{P}(e, \bar{w})$ as the principal's expected costs associated with inducing effort $e$. We have

$$
\begin{aligned}
& C^{P}(e, \bar{w})=\min _{(w, b, \bar{e})} \hat{w}(e, \bar{e})+b e \\
& \text { s.t. } \hat{w}(e, \bar{e})+b e-c(e) \geqq 0 \\
& e=\arg \max _{\widetilde{e} \geqq 0} \hat{w}(\widetilde{e}, \bar{e})+b \widetilde{e}-c(\widetilde{e}) \\
& 0 \leqq w \leqq \bar{w}
\end{aligned}
$$

where inequality (7) constitutes the participation constraint and (8) is the incentive compatibility constraint. The left-handed inequality in (9) reflects the agent's liquidity constraint while the right-handed inequality ensures that the implicit reward offer is credible.

\section{The compensation cost minimum}

Initially, take the bonus $b$ as given and denote by $e^{b}$ the effort level that would satisfy

$$
b=c_{e}\left(e^{b}\right)
$$

i.e. the effort sustainable by the bonus alone.

First consider the case where $\bar{e} \leqq e^{b}$. Obviously, whatever the salary $w$, the agent will implement $e^{b}$. Consequently, the principal should set $w=0$. Second consider the case where $\bar{e}>e^{b}$. The agent will either choose $e=e^{b}$ or $e=\bar{e}$. Suppose the agent selects $\widetilde{e}<\bar{e}$. Since he never receives the salary $w$, his optimal decision is characterized by (10) yielding $e=e^{b}$. Again in this case the principal should set $w=0$. Alternatively, suppose the agent were to select $\widetilde{e}>\bar{e}$. He receives the salary with certainty and the bonus with probability $\widetilde{e}$. This however cannot be optimal, since a marginal reduction in effort increases $b \widetilde{e}-c(\widetilde{e})$ but leaves the salary unaffected. Thus the only other possible solution is $e=\bar{e}$.

From the foregoing, the principal's cost-minimization problem can be restated as

$$
\begin{gathered}
C^{P}(e, \bar{w})=\min _{\left(w, e^{b}\right)} w+c_{e}\left(e^{b}\right) e \\
\text { s.t. }(9), w+c_{e}\left(e^{b}\right) e-c(e) \geqq 0
\end{gathered}
$$




$$
w+c_{e}\left(e^{b}\right) e-c(e) \geqq R\left(e^{b}\right)
$$

Inequality (11) is the agent's participation constraint and (12) constitutes the incentive compatibility constraint. The latter states that the agent is better off supplying effort $e$ rather than $e^{b}$. Since $R\left(e^{b}\right) \geqq 0$, (12) guarantees that the participation constraint (11) is always satisfied and can thus be ignored in the remaining analysis.

Insert figure 1 about here!

The transformation of problem (II) into problem (III) is illustrated graphically in figure 1. Suppose the principal offers a contract $(w, b, \bar{e})$. If the agent were to select $e=e^{b}$, determined by $c_{e}\left(e^{b}\right)=b$, he would not receive the implicit part of the wage. Geometrically, he would attain the rent represented by the distance $\overline{A B}$.

If the agent were to choose effort $\bar{e}$, he would receive the implicit part of the wage and the explicit bonus with probability $\bar{e}$. Geometrically, the rent is then measured by the distance $\overline{C D}$. Obviously, the agent will only choose effort $\bar{e}$ if $\overline{C D} \geqq \overline{A B}$. The figure also verifies the foregoing argument that the agent will never supply more effort than $\bar{e}$.

Cost-minimization on the part of the principal implies that he should set $w$ to satisfy $\overline{C D}=\overline{A B}$. Analytically, it means that the incentive compatibility constraint (12) is binding.

Figure 1 demonstrates the advantage of such a salary-augmented contract over a pure bonus contract. The former reduces the agent's rent from $R(e)=e c_{e}(e)-c(e)$ to $R\left(e^{b}\right)=e^{b} c_{e}\left(e^{b}\right)-c\left(e^{b}\right)$. As a result, the principal should attempt to keep $e^{b}$ as small as possible, i.e. to offer the smallest feasible bonus. There are two possible cases. If $w=c(e)$ is credible, the principal can reduce $e^{b}$ all the way to zero without violating (12). This is the obvious case where a pure reputational contract that extracts the entire rent is feasible.

However, if $\bar{w}<c(e)$, this solution is not feasible. The principal will therefore choose $w=\bar{w}$ and $e^{b}>0$ to satisfy the incentive compatibility condition

$$
\bar{w}+c_{e}\left(e^{b}\right) e-c(e)=R\left(e^{b}\right)
$$

This equation implicitly defines the function $e^{b}(e, \bar{w})$. An increase in $\bar{w}$ raises the RHS of the equation and allows for further reductions in $e^{b}$. In contrast, an increase 
in $e$ reduces the LHS and necessitates a higher $e^{b}$. Analytically,

$$
\begin{aligned}
& e_{e}^{b}(e, \bar{w})=\frac{c_{e}\left(e^{b}\right)-c_{e}(e)}{c_{e e}\left(e^{b}\right)\left[e^{b}-e\right]}>0, \\
& e_{\bar{w}}^{b}(e, \bar{w})=\frac{1}{c_{e e}\left(e^{b}\right)\left[e^{b}-e\right]}<0,
\end{aligned}
$$

since $e>e^{b}(e, \bar{w})$ in the compensation cost minimum.

We summarize the above conclusions in the following lemma.

Lemma 1: The minimum compensation cost of the principal associated with implementing the effort level $e$ is

$$
C^{P}(e, \bar{w})=\left\{\begin{array}{cl}
R\left(e^{b}(e, \bar{w})\right)+c(e) & , \text { if } \bar{w}<c(e) \\
c(e) & , \text { if } \bar{w} \geqq c(e)
\end{array},\right.
$$

For $\bar{w}<c(e)$, the lemma implies

$$
C_{e}^{P}(e, \bar{w})=c_{e}(e)+e^{b} \frac{c_{e}\left(e^{b}\right)-c_{e}(e)}{e^{b}-e}>c_{e}(e)>0
$$

and

$$
C_{\bar{w}}^{P}(e, \bar{w})=\frac{e^{b}}{e^{b}-e}<0 .
$$

Further, $C_{e}^{P}(e, \bar{w})=c_{e}(e)$ and $C_{\bar{w}}^{P}(e, \bar{w})=0$ if, and only if, $\bar{w} \geqq c(e)$ and, hence, $e^{b}(e, \bar{w})=0$. Finally, in the appendix we verify that $C_{e e}^{P}(e, \bar{w})>0$ and $C_{e \bar{w}}^{P}(e, \bar{w}) \leqq 0$ with strict inequality for $e^{b}(e, \bar{w})>0$.

\section{Implementing the optimal effort}

Now, recall that the maximally credible salary is endogenously determined in an infinitely repeated reputation game without discounting. If the principal breaches the contract at the end of a period, his one-time benefit is equal to the savings on salary $\bar{w}$. However, in every future period he can only offer a contract with purely explicit performance pay to the agent. 
From above, $\pi^{D}$ denotes the optimal per-period expected profit after deviation. Using (16), a contract implementing effort $e$ with both explicit and implicit performance pay is self-enforcing if

$$
\begin{aligned}
& \sum_{t=1}^{\infty} p^{t}\left[v(e)-C^{P}(e, \bar{w})-\pi^{D}\right] \geqq \bar{w} \\
& \Longrightarrow \phi\left[v(e)-C^{P}(e, \bar{w})-\pi^{D}\right] \geqq \bar{w}
\end{aligned}
$$

where $\phi=\frac{p}{(1-p)}$. Altogether, the principal's problem of maximizing his stream of expected profit in the infinitely repeated reputation game can be formulated as

$$
\begin{aligned}
\max _{(e, \bar{w})} \mathcal{L} & =v(e)-C^{P}(e, \bar{w}) \\
& +\eta\left[\phi\left[v(e)-C^{P}(e, \bar{w})-\pi^{D}\right]-\bar{w}\right]
\end{aligned}
$$

where $\eta$ denotes the Lagrange-multiplier.

The expected profit maximum is characterized by the following first-order conditions:

$$
\begin{gathered}
e:\left[v_{e}\left(e^{*}\right)-C_{e}^{P}\left(e^{*}, \bar{w}^{*}\right)\right]\left(1+\eta^{*} \phi\right)=0, \\
\bar{w}:-C_{\bar{w}}^{P}\left(e^{*}, \bar{w}^{*}\right)\left(1+\eta^{*} \phi\right)-\eta^{*}=0 .
\end{gathered}
$$

The superscript “*” indicates optimal values.

Proposition 1 Constraint (19) is binding in the optimization problem (20) if

$$
\phi\left[\pi^{F B}-\pi^{D}\right]<c\left(e^{F B}\right)
$$

where $\pi^{F B}=v\left(e^{F B}\right)-c\left(e^{F B}\right)$ denotes the first-best profit with $e^{F B}$ characterized by $v_{e}\left(e^{F B}\right)=c_{e}\left(e^{F B}\right)$. Given (23), the optimal explicit bonus is strictly positive.

Proof. Suppose that contrary to the assertion in the proposition (23) is satisfied, but that the constraint is not binding implying $\eta^{*}=0$. From (22), we conclude that $C_{\bar{w}}\left(\bar{w}^{*}, e^{*}\right)=0$ which requires $\bar{w}>c(e)$. This in turn implies $v_{e}\left(e^{*}\right)=c_{e}\left(e^{*}\right)-$ hence, the pure reputational contract implements the first-best effort level. Clearly, the principal then optimally sets $\bar{w}^{*}=c\left(e^{F B}\right)$. By insertion into (19) we obtain a contradiction, thus verifying that the constraint is indeed binding. 
Accordingly, $\eta^{*}>0$ implying $\left(1+\eta^{*} \phi\right)>0$, hence by $(22) e^{b}\left(\bar{w}^{*}, e^{*}\right)>0$ and $b^{*}=c_{e}\left(e^{b}\left(\bar{w}^{*}, e^{*}\right)\right)>0$.

Condition (23) defines a critical $\phi^{C}$ and a corresponding critical probability $p^{C}$ that the agent remains attached to the firm at the end of each period. For all $p<p^{C}$ the optimal contract always combines salary and bonus. In the remaining, we assume this to be the case. The resulting second-best solution is characterized by

$$
v_{e}\left(e^{*}\right)-C_{e}^{P}\left(e^{*}, \bar{w}^{*}\right)=0
$$

and

$$
\phi\left[v\left(e^{*}\right)-C^{P}\left(e^{*}, \bar{w}^{*}\right)-\pi^{D}\right]-\bar{w}^{*}=0 .
$$

It follows that

$$
\begin{gathered}
\left(\begin{array}{c}
\frac{d e^{*}}{d \phi} \\
\frac{d \bar{w}^{*}}{d \phi}
\end{array}\right)=\left(\begin{array}{cc}
v_{e e}\left(e^{*}\right)-C_{e e}^{P}\left(e^{*}, \bar{w}^{*}\right) & -C_{e \bar{w}}^{P}\left(e^{*}, \bar{w}^{*}\right) \\
0 & -\phi C_{\bar{w}}^{P}\left(e^{*}, \bar{w}^{*}\right)-1
\end{array}\right)^{-1}\left(\begin{array}{c}
0 \\
\frac{\bar{w}^{*}}{\phi}
\end{array}\right) \\
=-\frac{1}{\Delta}\left(\begin{array}{cc}
-\phi C_{\bar{w}}^{P}\left(e^{*}, \bar{w}^{*}\right)-1 & C_{e \bar{w}}^{P}\left(e^{*}, \bar{w}^{*}\right) \\
0 & v_{e e}\left(e^{*}\right)-C_{e e}^{P}\left(e^{*}, \bar{w}^{*}\right)
\end{array}\right)\left(\begin{array}{c}
0 \\
\frac{\bar{w}^{*}}{\phi}
\end{array}\right)
\end{gathered}
$$

where

$$
\begin{gathered}
\Delta=\left(-\phi C_{\bar{w}}^{P}\left(e^{*}, \bar{w}^{*}\right)-1\right)\left(v_{e e}\left(e^{*}\right)-C_{e e}^{P}\left(e^{*}, \bar{w}^{*}\right)\right) \\
=-\frac{1}{1+\eta^{*} \phi}\left(v_{e e}\left(e^{*}\right)-C_{e e}^{P}\left(e^{*}, \bar{w}^{*}\right)\right) .
\end{gathered}
$$

The second equality follows by insertion from the first-order condition (22). Consequently,

$$
\frac{d e^{*}}{d \phi}=-\frac{1}{\Delta} C_{e \bar{w}}^{P}\left(e^{*}, \bar{w}^{*}\right) \frac{\bar{w}^{*}}{\phi}
$$

and

$$
\frac{d \bar{w}^{*}}{d \phi}=-\frac{1}{\Delta}\left[v_{e e}\left(e^{*}\right)-C_{e e}^{P}\left(e^{*}, \bar{w}^{*}\right)\right] \frac{\bar{w}^{*}}{\phi} .
$$

Finally, we are interested in the response of the optimal explicit bonus. In this respect $b^{*}=c_{e}\left(e^{b}\left(\bar{w}^{*}, e^{*}\right)\right)$ implies

$$
\frac{d b^{*}}{d \phi}=c_{e e}\left(e^{b}\left(\bar{w}^{*}, e^{*}\right)\right)\left[e_{\bar{w}}^{b}\left(\bar{w}^{*}, e^{*}\right) \frac{d \bar{w}^{*}}{d \phi}+e_{e}^{b}\left(\bar{w}^{*}, e^{*}\right) \frac{d e^{*}}{d \phi}\right] .
$$


Proposition 2 Suppose that the probability that the agent remains within the firm in an upcoming period permanently increases. Then:

(a) the optimal effort supply $e^{*}$ increases;

(b) the optimal salary $\bar{w}^{*}$ increases;

(c) the optimal explicit bonus $b^{*}$ decreases.

Proof. Due to $\eta^{*}>0$ and, as shown in the appendix, $C_{e e}^{P}\left(e^{*}, \bar{w}^{*}\right)>0$, we have $\Delta>0$.

(a) The appendix also shows that $C_{e \bar{w}}^{P}\left(e^{*}, \bar{w}^{*}\right)<0$. Hence, the expression (28) is positive.

(b) Since $v_{e e}\left(e^{*}\right)-C_{e e}^{P}\left(e^{*}, \bar{w}^{*}\right)<0$, the expression (29) is positive.

(c) Having established (a) and (b), (30) can be rewritten as

$$
\begin{gathered}
\frac{d b^{*}}{d \phi}=-\left[\frac{\bar{w}^{*}}{\Delta \phi\left(e^{b}\left(\left(\bar{w}^{*}, e^{*}\right)-e^{*}\right)\right.}\right] \\
\times\left[\left(c_{e}\left(e^{b}\left(\bar{w}^{*}, e^{*}\right)\right)-c_{e}\left(e^{*}\right)\right) C_{e \bar{w}}^{P}\left(e^{*}, \bar{w}^{*}\right)+v_{e e}\left(e^{*}\right)-C_{e e}^{P}\left(e^{*}, \bar{w}^{*}\right)\right]<0 .
\end{gathered}
$$

The sign follows because $\left(c_{e}\left(e^{b}\left(\bar{w}^{*}, e^{*}\right)\right)-c_{e}\left(e^{*}\right)\right) C_{e \bar{w}}^{P}\left(e^{*}, \bar{w}^{*}\right)-C_{e e}^{P}\left(e^{*}, \bar{w}^{*}\right)<0$ as can be easily verified. ${ }^{7}$

The impact of a variation in $p$ is rather straightforward. A higher $p$ increases the credible maximum implicit reward associated with contract compliance. From (15), this allows a reduction in $e^{b}\left(\bar{w}^{*}, e^{*}\right)$ and the corresponding rent. Thus, the results (b) - (c) of the proposition reflect the principal's enhanced possibility to minimize his total compensation costs associated with inducing effort $e$. This in turn decreases the principal's marginal cost of implementing effort inducing her to choose a higher optimal effort level. Obviously, given (14), the increase in effort causes second-order effects. The proposition simply verifies that the primary effects dominate.

\footnotetext{
${ }^{7}$ In particular, note that $e_{e}^{b}(\bar{w}, e)=\left(c_{e}\left(e^{b}(\bar{w}, e)\right)-c_{e}(e)\right) e_{\bar{w}}^{b}(\bar{w}, e)$.
} 


\section{Conclusions and discussion}

The current study integrates the repeated game approach to implicit contracts and the analysis of explicit bonus rules based on performance evaluation to determine the optimal structure of the compensation scheme for the average white-collar employee. Previous studies by Baker et al. (1994) and Pearce and Stacchetti (1998) have emphasized optimal risk-shifting vis-a-vis the uncertainty of a possibly distorted performance signal or optimal income-smoothing over time, respectively. In contrast, the mechanism discussed above is associated with the principal's possibility to minimize a liquidity-constrained agent's rent.

Given a sufficiently low probability that the agent remains attached to his firm after each period, the optimal contract always entails both an implicit incentive salary and an explicit bonus rule. If this probability increases, the principal can reduce the agent's rent which is derived from the explicit bonus. Hence, the optimal salary and the optimal effort supply increase, while the explicit bonus decreases. Generally, the analysis implies that the share of bonus pay in total income decreases if, due to a more effective reputation mechanism, the optimal effort supply increases. The explicit bonus only substitutes imperfectly for reputational incentives that are insufficient to implement the first-best. Consequently, less variable bonus income reflects ceteris paribus more effective effort incentives.

The current analysis can be easily extended to incorporate the possibility of random productivity shocks. Suppose that in every period $t$ revenue is given by $v_{t}=\alpha_{t} v\left(e_{t}\right)$ where $\alpha_{t}$ constitutes a random variable with support $(0, \infty), E\left\{\alpha_{t}\right\}=1$, and $\operatorname{cov}\left(\alpha_{t}, \alpha_{\tau}\right)=0$ for $t \neq \tau$. The constraint (19) can then be restated in terms of expected values. Due to the assumption that the shocks are uncorrelated over time, the corresponding implicit reward $\bar{w}^{*}$ does not depend on the realizations of $\alpha_{t}$. In contrast, the first-order condition with respect to the effort supply in period $t$ yields $\alpha_{t} v_{e}\left(e_{t}^{*}\right)=C_{e}^{P}\left(e_{t}^{*}, \bar{w}^{*}\right)$. Comparative static analysis yields that optimal period $t$ effort is increasing in $\alpha_{t}$. Due to $b_{t}^{*}=c_{e}\left(e^{b}\left(\bar{w}^{*}, e_{t}^{*}\right)\right)$, it also implies that boni are increasing in the productivity shocks.

Given our framework, a statistician gathering data on productivity, effort, and boni would find a positive correlation between these variables. However, it would obviously be wrong to conclude that increasing bonus pay improves long-run profits. 
This finding questions some of the common wisdom found in textbooks as well as empirical investigations - in particular, the recent studies of performance pay for rank and file managers by Aggarval and Samwick (2003) and Rayton (2003).

The current analysis emphasizes that firms should generally avoid to imitate incentive schemes observed in contracting environments which do not exactly match their own. For instance, given the difference in unemployment rates between Europe and the US, it also appears plausible that firm-specific turnover rates are lower in Europe. This fact would explain the observed difference in incentive schemes between Europe and the US. Hence, "importing" US-type high-powered incentive schemes to European firms might well reduce long-run profits.

Finally, our study underlines the danger of misperceiving the implicit reward nature of the salary in firm-employee disputes. In particular, if labor courts rule that based on customary rights employees are entitled to a salary if such has been regularly paid in the past, the implicit reward structure would be destroyed. Firms would be forced to respond by increasing the share of variable income using explicit boni. Again, this would reduce long-run profits and effort.

\section{References}

Aggarval, Rajesh K. and Andrew A. Samwick. (2003). Performance Incentives within Firms: The Effect of Managerial Responsibility. Journal of Finance, vol. 58, p. 1613- 1649.

Baker, George P., Michael C. Jensen, and Kevin J. Murphy. (1988). Compensation and Incentives: Practise versus Theory. Journal of Finance, vol. 43, p. 593-616.

Baker, George P., Robert Gibbons, and Kevin J. Murphy. (1994). Subjective Performance Measures in Optimal Incentive Contracts. Quarterly Journal of Economics, vol. 109, p. 1125-1156.

Bull, Clive. (1987). The Existence of Self-Enforcing Implicit Contracts. Quarterly Journal of Economics, vol. 102, p. 147-159.

Demougin, Dominique and Claude Fluet. (2001). Monitoring versus Incentives. European Economic Review, vol. 45, p. 1741-1764. 
Ittner, Christopher D., David F. Larcker, and Marshall W. Meyer. (2003). Subjectivity and the Weighting of Performance Measures: Evidence from a Balanced Scorecard. Accounting Review, vol. 78, p. 725-758.

Levin, Jonathan. (2003). Relational Incentive Contracts. American Economic Review, vol. 93 , p. $835-847$.

MacLeod, W. Bentley. (2003). Optimal Contracting with Subjective Evaluation. American Economic Review, vol. 93, p. 216-240.

MacLeod, W. Bentley and James M. Malcolmsen. (1989). Implicit Contracts, Incentive Compatibility, and Involuntary Unemployment. Econometrica, vol. 57, p. 447-480.

MacLeod, W. Bentley and Daniel Parent. (1999) Jobs Characteristics and the Form of Compensation. Research in Labor Economics, vol. 18, p. 177-242.

Pearce, David G. and Ennio Stacchetti. (1998). The Interaction of Implicit and Explicit Contracts in Repeated Agency. Games and Economic Behavior, vol. 23, p. 75-96.

Prendergast, Canice. (1999). The Provision of Incentives in Firms. Journal of Economic Literature, vol. 23, p. 7-63.

Rayton, Bruce A. (2003). The Residual Claim of Rank and File Employees. Journal of Corporate Finance, vol. 9, p. 129-148. 


\section{Appendix}

From (18)

$$
\begin{aligned}
C_{e \bar{w}}^{P}(e, \bar{w}) & =\frac{1}{\left(e-e^{b}((\bar{w}, e))^{2}\right.}\left(e^{b}(\bar{w}, e)-e e_{e}^{b}(\bar{w}, e)\right) \\
& =\frac{1}{\left(e-e^{b}(\bar{w}, e)\right)^{2}}\left(e^{b}(\bar{w}, e)-e \frac{c_{e}(e)-c_{e}\left(e^{b}(\bar{w}, e)\right)}{c_{e e}\left(e^{b}(\bar{w}, e)\right)\left(e-e^{b}(\bar{w}, e)\right)}\right)<0 .
\end{aligned}
$$

To see this notice that $e^{b}<e$ and $e_{e}^{b} \geqq 1$ by the convexity of $c_{e}$.

Also, from (17)

$$
\begin{gathered}
C_{e e}^{P}(e, \bar{w})=c_{e e}(e)+e_{e}^{b}(\bar{w}, e) \frac{c_{e}\left(e^{b}(\bar{w}, e)\right)-c_{e}(e)}{e^{b}(\bar{w}, e)-e} \\
+\frac{e^{b}(\bar{w}, e)}{\left[e^{b}(\bar{w}, e)-e\right]^{2}}\left\{\left[c_{e}\left(e^{b}(\bar{w}, e)\right)-c_{e}(e)-c_{e e}(e)\left(e^{b}(\bar{w}, e)-e\right)\right]\right. \\
\left.+e_{e}^{b}(\bar{w}, e)\left[c_{e}(e)-c_{e}\left(e^{b}(\bar{w}, e)\right)+c_{e e}\left(e^{b}(\bar{w}, e)\right)\left(e^{b}(\bar{w}, e)-e\right)\right]\right\}
\end{gathered}
$$

where the separate terms have been arranged such that each one of them can be verified to be positive. In the second and third line of the equation, just notice that the elements of the squared bracket are Taylor-expansions. Again, the respective signs follow from the convexity of $c_{e}$. 


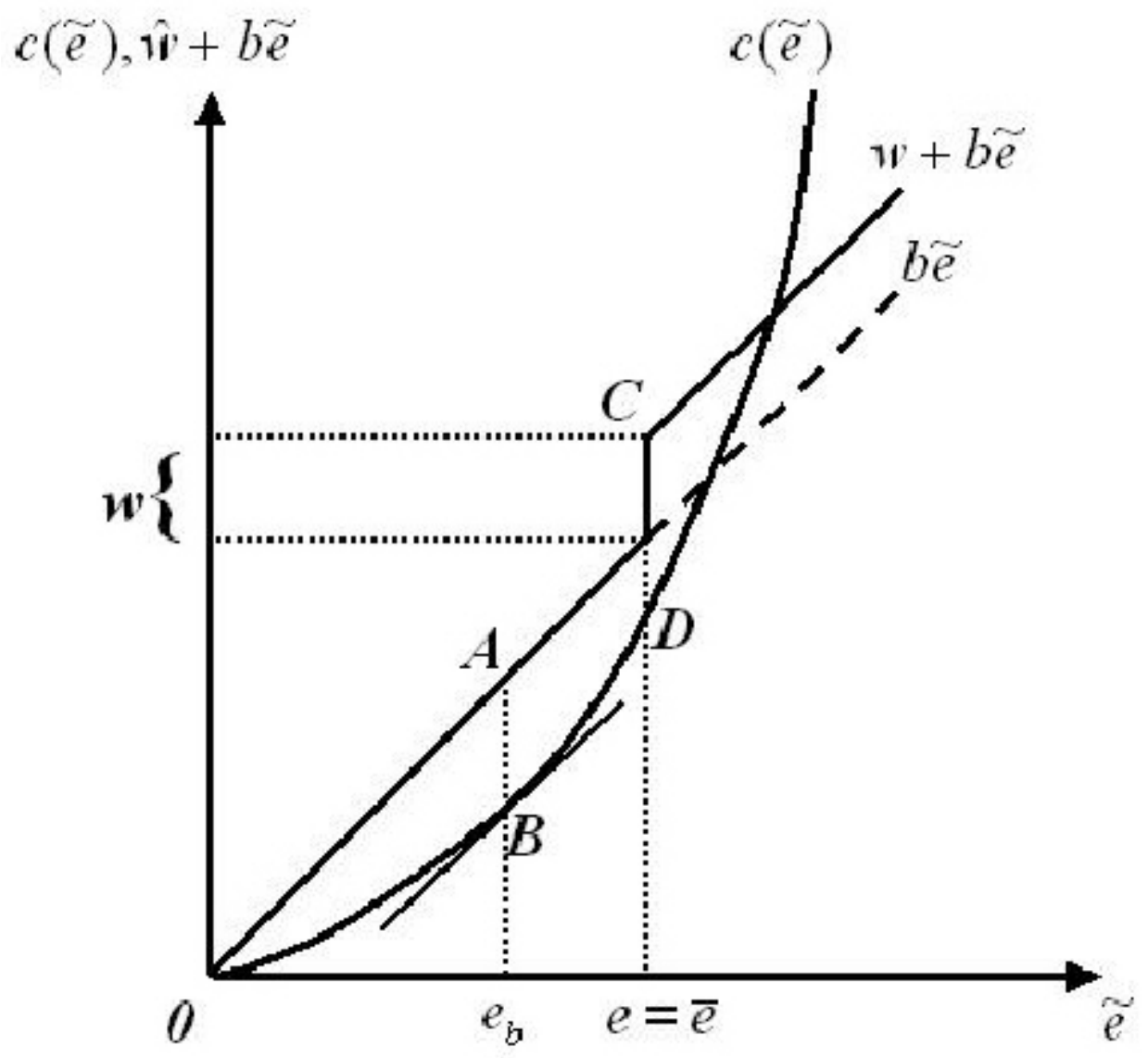

Figure 1: The interplay between explicit and implicit incentives in the cost function 